\title{
Downregulation of BRG-1 repressed expression of CD44s in cervical neuroendocrine carcinoma and adenocarcinoma
}

\author{
Kuan-Ting Kuo ${ }^{1, *}$, Cher-Wei Liang ${ }^{1, *}$, Chen-Hsiang Hsiao ${ }^{1}$, Ching-Hung Lin², \\ Chi-An Chen ${ }^{3}$, Bor-Ching Sheu ${ }^{3}$ and Ming-Chieh Lin ${ }^{1}$
}

${ }^{1}$ Department of Pathology, National Taiwan University Hospital, Medical College, National Taiwan University, Taipei, Taiwan; ${ }^{2}$ Department of Oncology, National Taiwan University Hospital, Medical College, National Taiwan University, Taipei, Taiwan and ${ }^{3}$ Department of Obstetrics and Gynecology, National Taiwan University Hospital, Medical College, National Taiwan University, Taipei, Taiwan

\begin{abstract}
Neuroendocrine carcinomas of the uterine cervix are rare tumors with early metastases, highly aggressive clinical behavior, and poor clinical outcome. Several adhesion molecules like cadherins have been tested in an attempt to explain their unique characteristics. Cluster differentiation 44 (CD44) is a widely expressed cell surface glycoprotein that serves as an adhesion molecule in cell-to-substrate and cell-to-cell interaction. We have examined the expression of the standard CD44 (CD44s) by immunohistochemical stains in the paraffinembedded cervical neoplasm tissue of 17 cases of primary cervical neuroendocrine carcinoma, 28 cases of cervical adenocarcinoma, and 50 cases of cervical squamous cell carcinoma. Loss of CD44s expression was found in 16 of 17 neuroendocrine carcinomas, 14 of 28 adenocarcinomas, and three of 50 squamous cell carcinomas. The differences were statistically significant. We also examined immunohistochemically the expression of the BRG-1 subunit of the SWI-SNF complex, which has been reported to regulate the expression of CD44 in all cases. Loss of BRG-1 expression was observed in 12/16, 6/14, and 1/3 CD44s-negative neuroendocrine carcinomas, adenocarcinomas, and squamous cell carcinomas, respectively. This study suggests that loss of the CD44s molecule may imply special biological behaviors of cervical neuroendocrine carcinomas, and loss of expression of BRG-1 may contribute to this.

Modern Pathology (2006) 19, 1570-1577. doi:10.1038/modpathol.3800687; published online 22 September 2006
\end{abstract}

Keywords: CD44s; BRG-1; cervical cancer; neuroendocrine carcinoma

Cluster differentiation 44 (CD44) is a family of widely distributed transmembrane glycoproteins. It is encoded by a gene that maps to chromosome 11 and contains more than 20 exons and is involved in cell-cell and cell-matrix interaction. ${ }^{1,2}$ Ten exons between 5 and 15 are called variant exons and are alternatively spliced with various combinations. The standard isoform (CD44s) is the smallest form of CD44, which does not contain the epitope encoded by variant exons and plays an important

Correspondence: Dr M-C Lin, MD, Department of Pathology, National Taiwan University Hospital, 7, Chung Shan South Road, Taipei 10001, Taiwan.

E-mail: mlin@ha.mc.ntu.edu.tw

*These authors equally contributed to this work.

Received 27 March 2006; revised 27 July 2006; accepted 31 July 2006; published online 22 September 2006 role in lymphocyte homing, tissue regeneration, and tumor cell dissemination. ${ }^{1,3-6}$

A role for CD44 in tumor progression has been documented in numerous clinical and experimental studies. $^{7,8}$ Aberrant expression of some forms of CD44 can enhance metastasis and tumor growth both in vitro and in vivo. ${ }^{9-11}$ It is believed that CD44 expression in some tumors increases as the tumor becomes more proliferative and invasive. ${ }^{7}$ In contrast to studies that correlate CD44 overexpression with cancer progression, several reports also indicate that loss of CD44 expression may contribute to tumorigenesis. ${ }^{12}$ Specifically, it has been shown that loss of CD44 in some kinds of tumor, like cervical carcinomas, neuroblastomas, prostate carcinomas, melanomas, and small cell lung carcinomas, correlates with increased aggressiveness of these tumors. ${ }^{13-17}$ It has been hypothesized that the loss of 
CD44 may facilitate the abrogation of epithelialmesenchymal interactions in some tumors, leading to a more metastatic phenotype. ${ }^{12}$ Recently, a positive regulator of CD44, BRG-1, has been identified as a critical regulator of CD44 expression. ${ }^{18}$

BRM (Brahma) and its homolog, BRG-1 (Brahma related gene 1), are the components of the SWI/SNF ATPases that regulate gene transcription through ATPase-dependent remodeling of chromatin. ${ }^{19-22}$ BRG-1 has been shown to inhibit the transcription of a subset of genes involved in cancer, such as Cyclin A and c-fos. ${ }^{23-26}$ It can also facilitate estrogen receptor, glucocorticoid receptor, c-Myc, and BRCA1-mediated transcription. ${ }^{27-31}$ Recent reports demonstrate that the absence of BRG-1 correlates with a lack of CD44 expression in several cell lines while the reintroduction of BRG-1 restores the expression of CD44 in BRG-1-deficient cells, suggesting that BRG-1 regulates basal CD44 levels. ${ }^{18}$

Neuroendocrine tumors of the uterine cervix can be divided into carcinoid, atypical carcinoid, small cell carcinoma, and large cell neuroendocrine carcinoma according to histopathologic pictures, nuclear atypia, and mitotic figures, etc. ${ }^{32}$ Small cell carcinoma of the cervix has long been recognized as an uncommon, but highly aggressive subset of uterine cervical neoplasm, and similar biologic behavior can be observed in the large cell neuroendocrine carcinoma. ${ }^{32-38}$ The neuroendocrine carcinoma mentioned here refers to small cell carcinoma and large cell neuroendocrine carcinoma. Although expression of CD44s and its isoforms in cervical lesions has been reported by several sources,${ }^{39-41}$ the total case number is limited and no published paper discusses the expression of CD44 isoforms in cervical neuroendocrine tumors or the different expression rates of major cancer types. In contrast, loss of CD44s in nearly all cases of small cell lung cancer has been reported, and different expression rates are noted between small cell lung cancer and non-small cell carcinoma. ${ }^{42}$

In this study, 17 cases of primary cervical neuroendocrine carcinoma, 28 cases of cervical adenocarcinoma, and 50 cases of cervical squamous cell carcinoma were tested for CD44s expression by immunohistochemistry. Using the same method, we also tested for BRG-1 expression in order to prove the theory that BRG-1 regulates CD44 levels, a theory that has already been proven in cell lines but not in tumor tissue.

\section{Materials and methods}

\section{Patients and Specimens}

Formalin-fixed paraffin-embedded tissue blocks were obtained from 50 patients (41-62 years old, median age 51) with squamous cell carcinoma, 28 patients (37-61 years old, median age 47) with adenocarcinoma and 17 patients (31-52 years old, median age 39) with neuroendocrine carcinoma.
The histologic types were determined via histologic examination of H\&E stained tissue sections. All the cases of neuroendocrine carcinoma were immunoreactive for either Chromogranin A or Synaptophysin and included one case of large cell neuroendocrine carcinoma and 16 cases of small cell carcinoma. All patients underwent total surgical excision of their lesions at the Department of Obstetrics and Gynecology, National Taiwan University Hospital, Taipei, Taiwan, during the period between August 1994 and December 2005.

\section{Immunohistochemistry}

All specimens were formalin-fixed, paraffin-embedded, cut at $5-\mu \mathrm{m}$ thick sections, then deparaffinized, rehydrated, and underwent antigen retrieval (Trilogy, Cell Marque, Hot Springs, AR, USA; autoclaved, $10 \mathrm{~min}$ ). The sources of the primary antibodies were CD44s (neomarker; 156-3C11), BRG-1 (Santa Cruz; SC-10768), Chromogranin A (Dako; DAK-A3), and Synaptophysin (Cell Marque). Staining was performed using the Ventana autostainer (DAB detection kit, iView, Ventana Medical Systems, SA, USA). Cases in which more than $10 \%$ of cancer cells expressed the markers were thought to be positive.

\section{Statistical Analysis}

The Mann-Whitney $U$-test was used to evaluate statistical differences in age distribution between cancer types. The $\chi^{2}$ with Fisher's exact test was used to evaluate statistical differences of CD44s and BRG-1 positive rates between different histologic cancer types. A $P$-value less than 0.05 was considered significant.

\section{Results}

\section{Patients}

The neuroendocrine carcinoma patients (31-52 years old, median age 39) were statistically younger than the adenocarcinoma (37-61 years old, median age 47) and squamous cell carcinoma (41-62 years old, median age 51) patients, but the latter two had no statistically significant age difference.

\section{CD44s}

Normal squamous epithelium, which expressed CD44s stronger in the stratum basale and spinosum and contained diffusely CD44s-positive stromal and hematologic cells, served as positive control (Figure 1a). Lack of immunoreactivity for CD44s or weak staining in the basal portion was noted in the normal endocervical glands (Figure 1b). Fourteen of $28(50 \%)$ adenocarcinoma cases (Figure 3a and b), 


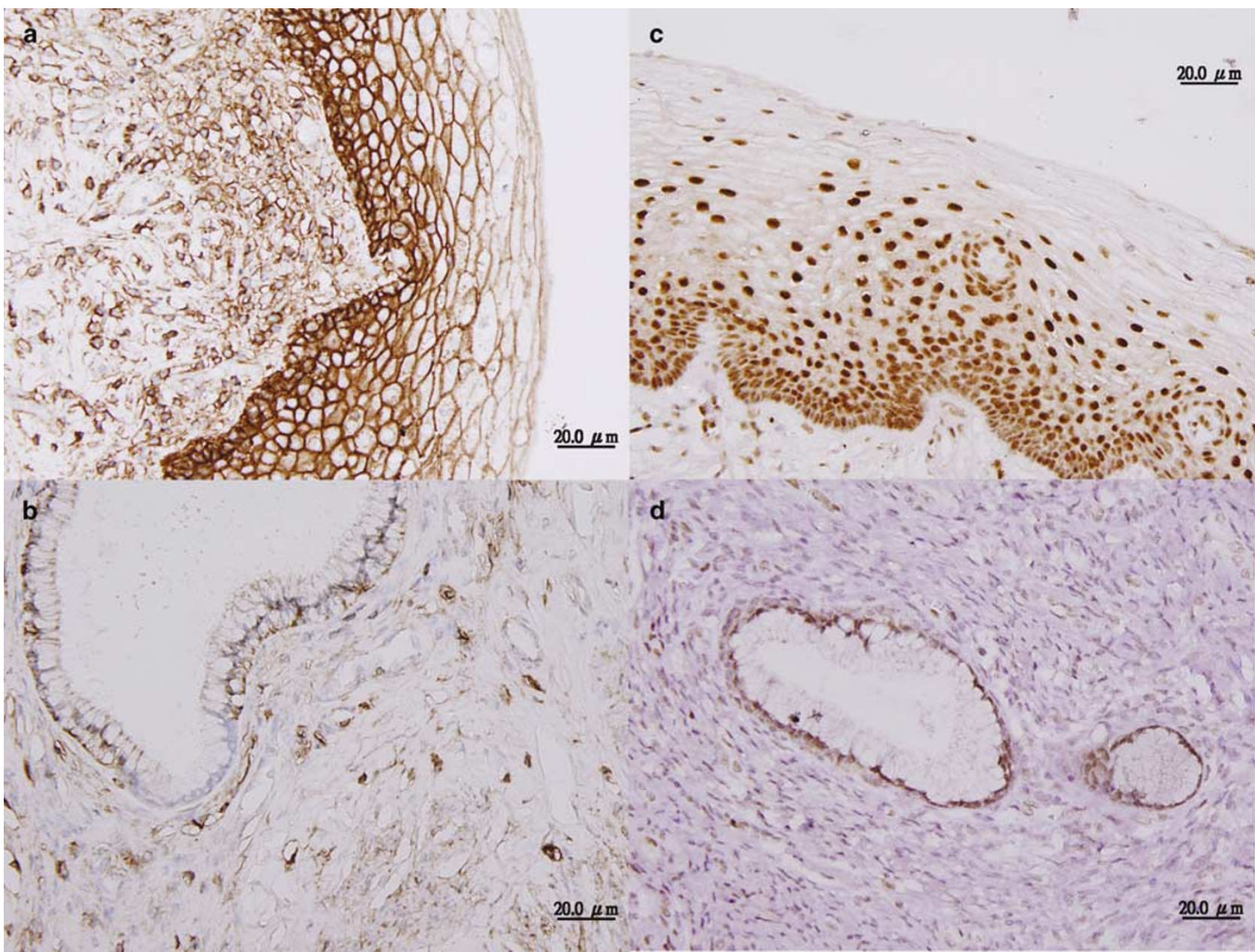

Figure 1 Immunohistochemical satins of CD44s and BRG-1 in normal exo- and endocervical mucosa $(\times 400)$. (a) Normal exocervical squamous epithelium; stromal and hematologic cells express CD44s. (b) Lack of immunoreactivity for CD44s or weak staining in the basal portion in the normal endocervical glands. (c) Normal exocervical squamous epithelium showed nuclear staining of BRG-1. (d) Normal endocervical glands show nuclear staining of BRG-1.

16 of $17(94.1 \%)$ neuroendocrine carcinoma cases (Figure $4 \mathrm{a}$ and $\mathrm{b})$, and 3 of $50(6 \%)$ squamous cell carcinoma (Figure 2a and b) cases did not express CD44s, and the loss rate of each group in comparison with either of the other two had statistical significance (Table 1).

\section{BRG-1}

Normal squamous epithelium (Figure 1c) and endocervical glands (Figure 1d) showed nuclear staining and served as positive control. Six of $28(21.43 \%)$ adenocarcinoma cases (Figure $3 \mathrm{c}$ and d), 12 of $17(70.59 \%)$ neuroendocrine carcinoma cases (Figure 4c and d), and 1 of $50(2 \%)$ squamous cell carcinoma (Figure 2c and d) cases did not express BRG-1. Statistical significances of the loss rates were recorded in Table 2. All BRG-1 negative cases were nonreactive for CD44s as well, and that was statistically significant $(P<0.001)$.

\section{Discussion}

Several reports have focused on CD44s and expression of its isoforms in uterine cervical cancer and precancerous lesions, but the expression rates of CD44s in various histologic types of cervical cancers have so far been inconclusive. None of these reports included an adequate number of cases of neuroendocrine carcinoma. ${ }^{39-41,43}$ In our study, the expression rates of CD44s are statistically higher in squamous cell carcinomas, as compared to adenocarcinomas and neuroendocrine carcinomas, but different results exist. ${ }^{39,41}$ The CD44s expression rates in our squamous cell carcinoma cases are slightly higher than that of the Faleiro-Rodrigues' series, ${ }^{41}$ but our adenocarcinoma cases have CD44s expression rates lower than that of Lu's series. ${ }^{39} \mathrm{In}$ Saegusa's series, the average immunoreactivity scores for CD44s were significantly higher in squamous cell carcinoma cases than in adenocarcinoma cases, a result which is similar to ours. ${ }^{43} \mathrm{We}$ 


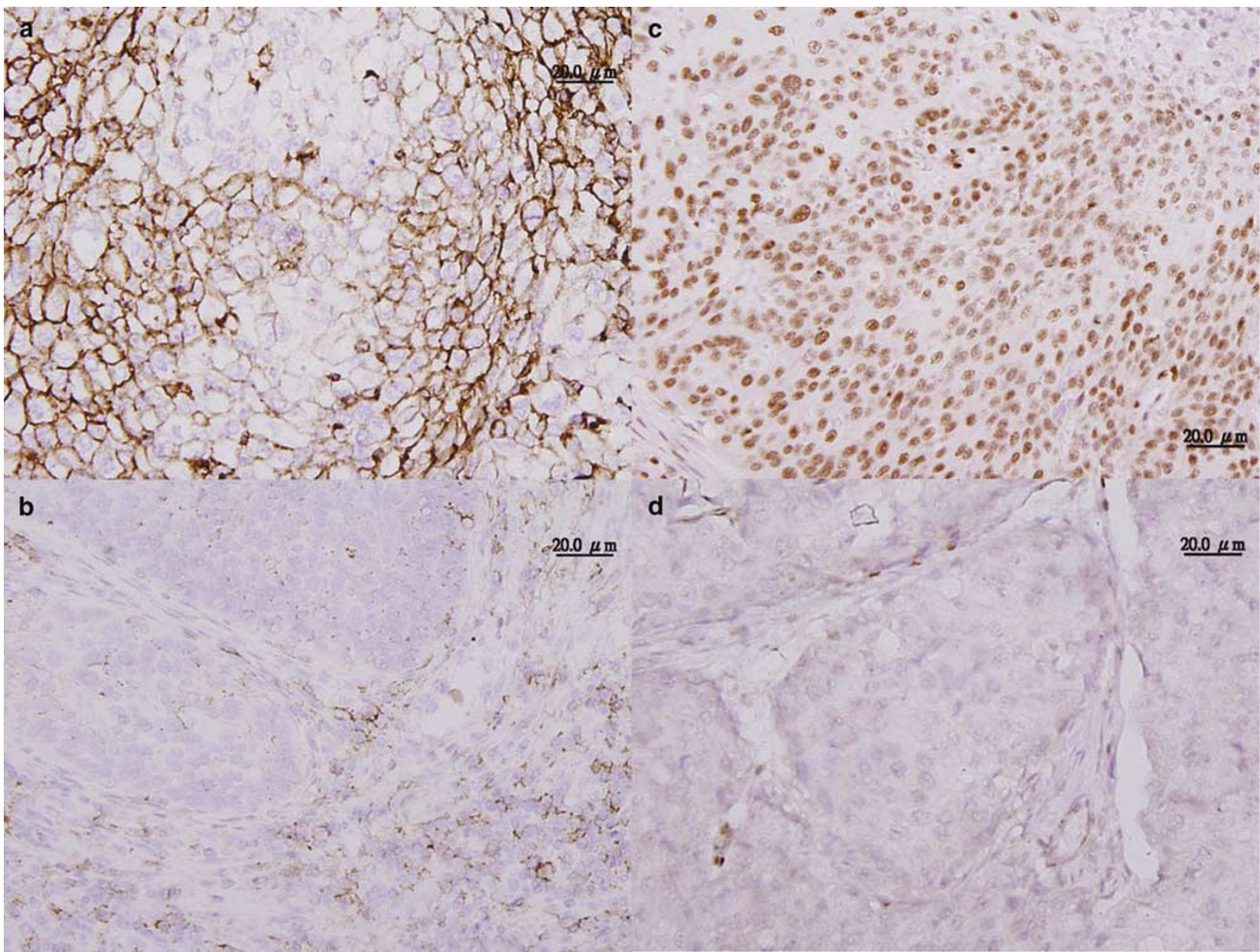

Figure 2 Immunohistochemical stains of CD44s and BRG-1 in cases of cervical squamous cell carcinoma $(\times 400)$. (a) CD44s-positive squamous cell carcinoma. (b) CD44s-negative squamous cell carcinoma. (c) BRG-1-positive squamous cell carcinoma. (d) BRG-1-negative squamous cell carcinoma.

Table 1 Comparison of CD44s loss rates between different cancer types

\begin{tabular}{lcc}
\hline Cancer type (case number) & CD44s loss rate & P-value \\
\hline Neuroendocrine carcinoma $(n=17)$ & $\begin{array}{c}16 / 17(94.1 \%) \\
\text { Adenocarcinoma }(n=28)\end{array}$ & 0.003 \\
& $14 / 28(50 \%)$ & \\
Neuroendocrine carcinoma $(n=17)$ & $16 / 17(94.1 \%)$ & $<0.001$ \\
Squamous cell carcinoma $(n=50)$ & $3 / 50(6 \%)$ & \\
Adenocarcinoma $(n=28)$ & $14 / 28(50 \%)$ & $<0.001$ \\
Squamous cell carcinoma $(n=50)$ & $3 / 50(6 \%)$ & \\
\hline
\end{tabular}

think this may be due to different criteria for positivity and different cancer subtype differentiation. In Lu's study, single-cell positivity was acknowledged; in our series, however, only cases in which more than $10 \%$ of the cancer cell population expressed the markers were recorded as being positive. In an earlier study, reactivity for CD44s was lacking in normal glandular type epithelium and in adenocarcinomas composed entirely of well-differentiated mucin-producing glands. Well-differentiated squamous cell carcinomas were consistently positive for CD44s but the results of poorly differentiated squamous cell carcinomas were variable. ${ }^{44}$

Neuroendocrine carcinoma of the cervix has long been recognized as an uncommon, but highly aggressive subset of uterine cervical neoplasm with characteristics such as young patient age, early metastasis, and particularly poor prognosis compared with other phenotypes. ${ }^{32-38}$ In our series, the age distribution of the neuroendocrine carcinoma group is significantly younger than the adenocarcinoma and squamous cell carcinoma groups. Twelve of the 17 neuroendocrine carcinoma cases had adequate clinical data showing that nine of the 12 cases suffered early metastasis to areas including bone, lung, brain, adrenal gland, and regional lymph nodes, etc. Eight of these nine cases had a loss of expression of CD44s. As in cervical adenocarcinomas and squamous cell carcinomas, high-risk types of HPV, especially HPV18, have been implicated in the carcinogenesis of cervical neuroendocrine 


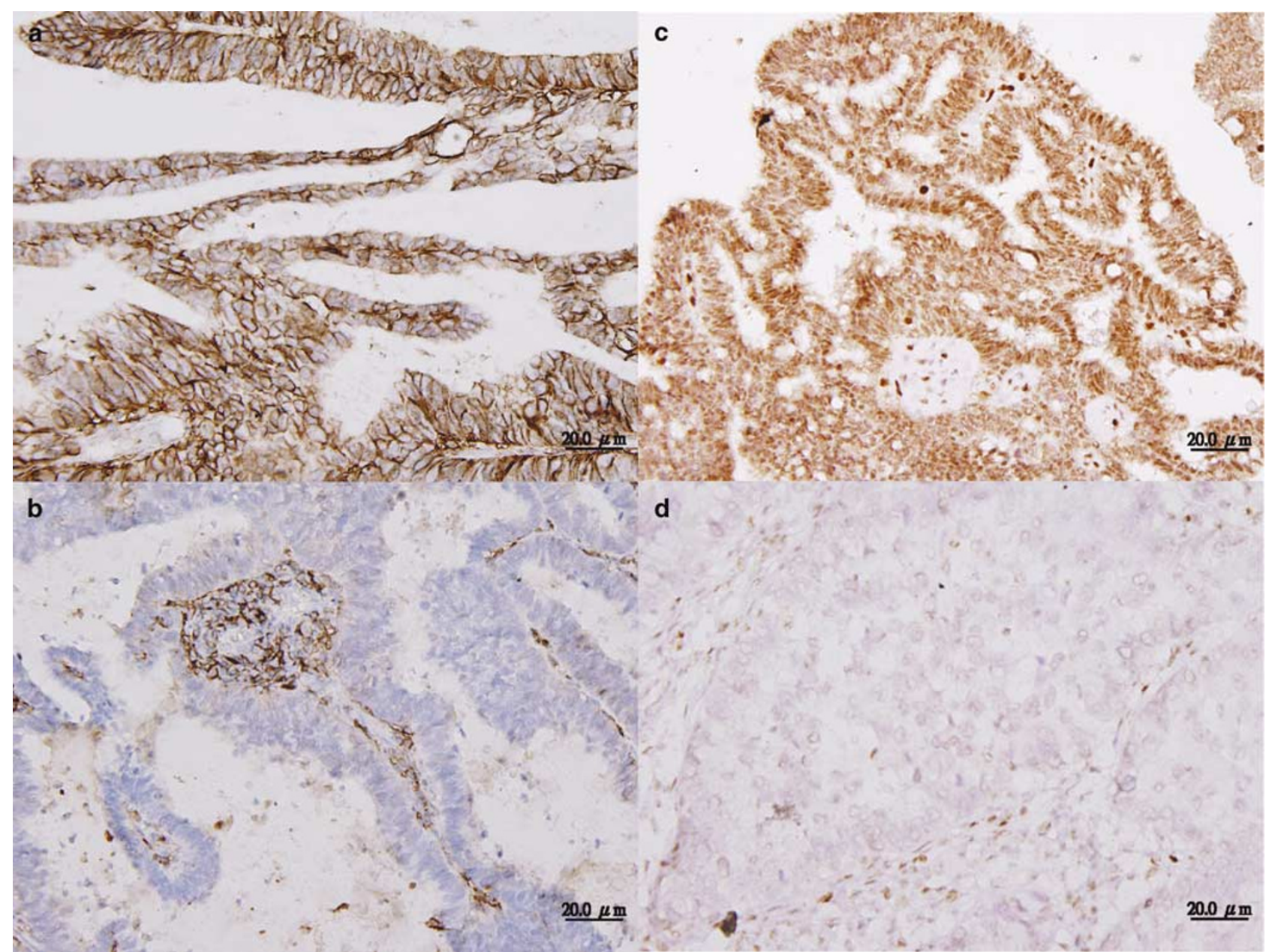

Figure 3 Immunohistochemical satins of CD44s and BRG-1 in cases of cervical adenocarcinoma $(\times 400)$. (a) CD44s-positive adenocarcinoma. (b) CD44s-negative adenocarcinoma. (c) BRG-1-positive adenocarcinoma. (d) BRG-1-negative adenocarcinoma.

carcinomas. ${ }^{45}$ In our series, HPV18 can be detected in all 17 cases of neuroendocrine carcinoma. Ours is the first report on the CD44s expression rate in cervical neuroendocrine carcinoma. The strikingly low-expression rate in our series (1/17) is similar to that of pulmonary small cell carcinomas, which are also clinically aggressive, prone to early metastases, and exclusively negative for CD44s. ${ }^{42}$ These two kinds of tumors, although from different organs, share similar neuroendocrine differentiation and clinical outcomes. Loss of CD44s expression may be associated with their cell behaviors.

The CD44 gene contains more than twenty exons. $^{1,2}$ When transcription into mRNA occurs, some of these exons are always included (constitutive exons), whereas the remainder may be included or excluded (alternative or variant exons). ${ }^{46}$ The surface glycoprotein CD44 adopts many isoforms that mediate vastly different functions in various types of normal cells. In the lymphocytes, for example, the interaction between CD44 and endothelial venules of lymph nodes is important to the homing passage of these cells. ${ }^{47}$ The role of CD44 expression in cancer cell biology is still not clear. ${ }^{7}$
Its influence on cancer cells from different origins varies. $^{7,8,48}$ In some cancers, aberrant expression of CD44 is associated with advanced tumor progression and poor clinical outcome,,$^{7,8,48}$ but in cancers of other organs, the loss of CD44 expression correlates with increased metastatic potential. ${ }^{7}$

BRM and BRG-1 are components of the SWI/SNF ATPases that regulate gene transcription through ATPase-dependent remodeling of chromatin. ${ }^{19-22}$ SWI/SNF is a multimeric chromatin remodeling complex important for gene regulation. Like other proteins involved in gene regulation, the SWI/SNF complex is a potential target for disruption during neoplastic progression. In yeast, proper functioning of the complex requires the functional integrity of the subunits to be intact, and loss of any one component is sufficient to alter the function of the complex. ${ }^{49}$ Thus, disruption of the SWI/SNF complex by the loss of other subunits may also be associated with human tumorigenesis. Previous investigations have shown an evolving role for the mutually exclusive SWI/SNF ATPases, BRM and BRG1, in growth control and tumorigenesis. ${ }^{50}$ 


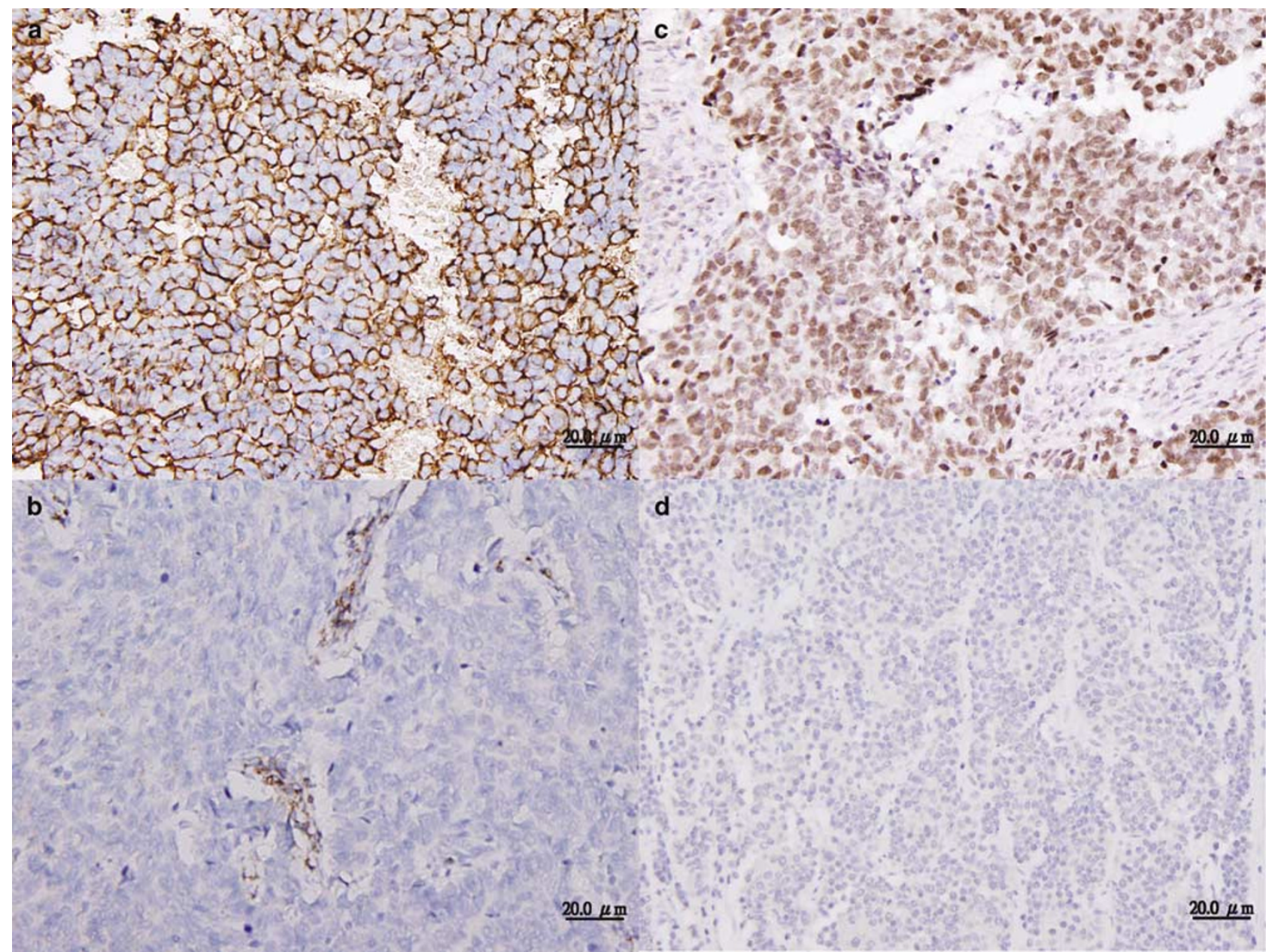

Figure 4 Immunohistochemical satins of CD44s and BRG-1 in cases of cervical neuroendocrine carcinoma. (a) CD44s-positive neuroendocrine carcinoma. (b) CD44s-negative neuroendocrine carcinoma. (c) BRG-1-positive neuroendocrine carcinoma. (d) BRG-1negative neuroendocrine carcinoma.

Table 2 Comparison of BRG-1 loss rates between different cancer types

\begin{tabular}{|c|c|c|}
\hline Cancer type (case number) & BRG-1 loss rate & P-value \\
\hline Neuroendocrine carcinoma $(n=17)$ & $12 / 17(70.59 \%)$ & 0.086 \\
\hline Adenocarcinoma $(n=28)$ & $6 / 28(21.43 \%)$ & \\
\hline Neuroendocrine carcinoma $(n=17)$ & $12 / 17(70.59 \%)$ & $<0.001$ \\
\hline Squamous cell carcinoma $(n=50)$ & $1 / 50(2 \%)$ & \\
\hline Adenocarcinoma $(n=28)$ & $6 / 28(21.43 \%)$ & 0.008 \\
\hline Squamous cell carcinoma $(n=50)$ & $1 / 50(2 \%)$ & \\
\hline
\end{tabular}

Although the mechanism for CD44 loss in some tumors is still not clear, according to a previous report, BRG-1 may play a critical role in regulating CD44 transcription. ${ }^{18}$ Reintroduction of BRG-1 restored CD44 expression in previous BRG-1-negative cell lines, whereas expression of dominant-negative BRG-1 inhibited CD44 production in other cell lines. These results indicate that BRG-1 is required for regulating CD44 expression, suggesting that loss of BRG-1 may contribute to the invasive and metastatic potential of certain cancers. ${ }^{18}$ Our study is the first attempt to prove this hypothesis using human primary cervical cancer tissues. Loss of expression of CD44s and BRG-1 occurred most frequently in the most aggressive histologic cervical cancer type, neuroendocrine carcinoma. Reisman's group demonstrated that loss of BRG-1/BRM in human lung cancer cell lines and primary lung cancers correlated with poor prognosis. ${ }^{51}$

Our experiment demonstrated different expression rates of CD44s and BRG-1 in primary cancer tissue of three important cervical cancer types. That the loss of expression of these two molecules occurred most frequently in the most aggressive histologic type, neuroendocrine carcinoma, may imply an association between aggressive cancer behavior and loss of these two molecules. Additional investigations are necessary to support this view.

\section{References}

1 Lesley J, Hyman R, Kincade PW. CD44 and its interaction with extracellular matrix. Adv Immunol 1993; 54:271-335. 
2 Screton GR, Bell MV, Jackson DG, et al. Genomic structure of DNA encoding the lymphocyte homing receptor $\mathrm{CD} 44$ reveals at least 12 alternatatively spliced exons. Proc Natl Acad Sci USA 1992;89: 12160-12164.

3 Gunthert U. CD44: a multitude of isoforms with diverse functions. Curr Top Microbiol Immunol 1993;184:47-63.

4 Haynes BF, Telen MJ, Hale LP, et al. CD44: a molecule involved in leukocyte adherence and T-cell activation. Immunol Today 1989;10:423-428.

5 Ponta H, Sleeman J, Dall P, et al. CD44 isoforms in metastatic cancer. Invasion Metastasis 1995;14:82-86.

6 Goodison S, Tarin D. Current status of CD44 variant isoforms as cancer diagnostic markers. Histopathology 1998;32:1-6.

7 Naot D, Sionov RV, Ish-Shalom D. CD44: structure, function, and association with the malignant process. Adv Cancer Res 1997;71:241-319.

8 Goodison S, Urquidi V, Tarin D. CD44 cell adhesion molecules. Mol Pathol 1999;52:189-196.

9 Gunthert U, Hofmann M, Rudy W, et al. A new variant of glycoprotein CD44 confers metastatic potential to rat carcinoma cells. Cell 1991;65:13-24.

10 Rudy W, Hofmann M, Schwartz-Albiez R, et al. The two major CD44 proteins expressed on a metastatic rat tumor cell line are derived from different splice variants: each one individually suffices to confer metastatic behavior. Cancer Res 1993;53:1262-1268.

11 Sleeman JP, Arming S, Moll JF, et al. Hyaluronateindependent metastatic behavior of CD44 variantexpressing pancreatic carcinoma cells. Cancer Res 1996;56:3134-3141.

12 Goodison S, Tarin D. Front. Bioscience (Online) 1998;3:E89-E109.

13 Kohlberger PD, Kieback DG, Bancher D, et al. Immunohistochemical detection of CD44 splice variant expression in premalignant lesions of the cervix and benign cervical epithelium. Gynecol Oncol 1997;66: 227-232.

14 Saegusa M, Machida D, Hashimura M, et al. CD44 expression in benign, premalignant, and malignant ovarian neoplasms: relation to tumour development and progression. J Pathol 1999;189:326-337.

15 Kallakury BV, Yang F, Figge J, et al. Decreased levels of CD44 protein and mRNA in prostate carcinoma. Correlation with tumor grade and ploidy. Cancer 1996;78:1461-1469.

16 Li H, Liu J, Hofmann M, et al. Differential CD44 expression patterns in primary brain tumours and brain metastases. Br J Cancer 1995;72:160-163.

17 Karjalainen JM, Tammi RH, Tammi MI, et al. Reduced level of CD44 and hyaluronan associated with unfavorable prognosis in clinical stage I cutaneous melanoma. Am J Pathol 2000;157:957-965.

18 Matthew WS, DeCristofaro MF, Banine F, et al. The BRG-1 subunit of the SWI/SNF complex regulates CD44 expression. J Biol Chem 2001;276:9273-9278.

19 Meraldi P, Lukas J, Fry AM, et al. Centrosome duplication in mammalian somatic cells requires E2F and Cdk2-cyclin A. Nat Cell Biol 1999;1:88-93.

20 Khavari PA, Peterson CL, Tamkun JW, et al. BRG1 contains a conserved domain of the SWI2/SNF2 family necessary for normal mitotic growth and transcription. Nature 1993;366:170-174.

21 Kwon H, Imbalzano AN, Khavari PA, et al. Nucleosome disruption and enhancement of activator binding by a human SW1/SNF complex. Nature 1994;370: $477-481$.

22 Imbalzano AN, Kwon H, Green MR, et al. Facilitated binding of TATA-binding protein to nucleosomal DNA. Nature 1994;370:481-485.

23 Zhang HS, Gavin M, Dahiya A, et al. Exit from G1 and $S$ phase of the cell cycle is regulated by repressor complexes containing HDAC-Rb-hSWI/SNF and RbhSWI/SNF. Cell 2000;101:79-89.

24 Strobeck MW, Knudsen KE, Fribourg AF, et al. BRG-1 is required for RB-mediated cell cycle arrest. Proc Natl Acad Sci USA 2000;97:7748-7753.

25 Trouche D, Le Chalony C, Muchardt C, et al. RB and hbrm cooperate to repress the activation functions of E2F1. Proc Natl Acad Sci USA 1997;94:1126811273.

26 Murphy DJ, Hardy S, Engel DA. Human SWI-SNF component BRG1 represses transcription of the c-fos gene. Mol Cell Biol 1999;19:2724-2733.

27 DiRenzo J, Shang Y, Phelan M, et al. BRG-1 is recruited to estrogen-responsive promoters and cooperates with factors involved in histone acetylation. Mol Cell Biol 2000;20:7541-7549.

28 Fryer CJ, Archer TK. Chromatin remodelling by the glucocorticoid receptor requires the BRG1 complex. Nature 1998;393:88-91.

29 de La Serna IL, Carlson KA, Hill DA, et al. Mammalian SWI-SNF complexes contribute to activation of the hsp70 gene. Mol Cell Biol 2000;20:2839-2851.

30 Cheng SW, Davies KP, Yung E, et al. c-MYC interacts with INI1/hSNF5 and requires the SWI/SNF complex for transactivation function. Nat Genet 1999;22: 102-105.

31 Bochar DA, Wang L, Beniya $\mathrm{H}$, et al. BRCA1 is associated with a human SWI/SNF-related complex: linking chromatin remodeling to breast cancer. Cell 2000;102:257-269.

32 Albores-Saavedra J, Gersell D, Gilks CB, et al. Terminology of endocrine tumors of the uterine cervix: results of a workshop sponsored by the College of American Pathologists and the National Cancer Institute. Arch Pathol Lab Med 1997;121:34-39.

33 Pisani P, Parkin DM, Ferlay J. Estimates of the worldwide mortality from eighteen major cancers in 1985. Implications for prevention and projections of future burden. Int J Cancer 1993;55:891-903.

34 Parker SL, Tong T, Bolden S, et al. Cancer statistics, 1996. CA Cancer J Clin 1996;46:5-27.

35 Parker SL, Tong T, Bolden S, et al. Cancer statistics, 1997. CA Cancer J Clin 1997;47:5-27.

36 Rrgan JW, Hamonic MJ, Wentz WB. Analytical study of the cells in cervical squamous-cell cancer. Lab Invest 1957;6:241-250.

37 Wentz WB, Reagan JW. Survival in cervical cancer with respect to cell type. Cancer 1959;12:384-388.

38 Gilks CB, Young RH, Gersell DJ, et al. Large cell neuroendocrine carcinoma of the uterine cervix: a clinicopathologic study of 12 cases. Am J Surg Pathol 1997;21:905-914.

39 Di L, Tawfik O, Pantazis C, et al. Altered expression of CD44 and variant isoforms in human adenocarcinoma of the endocervix during progression. Gynecol Oncol 1999;75:84-90.

40 Shimabukuro K, Toyama-Sorimachi N, Ozaki Y, et al. The expression patterns of standard and variant CD44 molecules in normal uterine cervix and cervical cancer. Gynecol Oncol 1997;64:26-34. 
41 Faleiro-Rodrigues C, Lopes C. E-cadherin, CD44 and CD44v6 in squamous intraepithelial lesions and invasive carcinomas of the uterine cervix: an immunohistochemical study. Pathobiology 2004;71: 329-336.

42 Ariza A, Mate JL, Isamat M, et al. Standard and variant CD44 isoforms are commonly expressed in lung cancer of the non-small cell type but not of the small cell type. J Pathol 1995;177:363-368.

43 Saegusa M, Hashimura M, Machida D, et al. Down-regulation of CD44 standard and variant isoforms during the development and progression of uterine cervical tumours. J Pathol 1999;187: 173-183.

44 Ylagan LR, Scholes J, Demopoulos R. Cd44: a marker of squamous differentiation in adenosquamous neoplasms. Arch Pathol Lab Med 2000;124:212-215.

45 Stoler MH, Mills SE, Gersell DJ, et al. Small-cell neuroendocrine carcinoma of the cervix. A human papillomavirus type 18-associated cancer. Am J Surg Pathol 1991;15:28-32.
46 Fox SB, Gatter KC, Jackson DG, et al. CD44 and cancer screening. Lancet 1993;342:548-549.

47 Jalkanen S, Steere AC, Fox RI, et al. A distinct endothelial cell recognition system that controls lymphocyte traffic into inflamed synovium. Science 1986;233:556-558.

48 Sherman L, Sleeman J, Dall P, et al. The CD44 proteins in embryonic development and in cancer. Curr Top Microbiol Immunol 1996;213:249-269.

49 Laurent BC, Treitel MA, Carlson M. Functional interdependence of the yeast SNF2, SNF5, and SNF6 proteins in transcriptional activation. Proc Natl Acad Sci USA 1991;88:2687-2691.

50 Bultman S, Gebuhr T, Yee D, et al. A Brg1 null mutation in the mouse reveals functional differences among mammalian SWI/SNF complexes. Mol Cell 2000;6:1287-1295.

51 David NR, Sciarrotta J, Wang W, et al. Loss of BRG1/ BRM in human lung cancer cell lines and primary lung cancers: correlation with poor prognosis. Cancer Res 2003;63:560-566. 